\title{
ПОДДЕРЖКА ПРИНЯТИЯ МАРКЕТИНГОВЫХ РЕШЕНИЙ НА ОСНОВЕ МЕТОДОВ НЕЧЕТКОГО УПРАВЛЕНИЯ
}

\section{SUPPORT FOR MARKETING DECISIONS BASED ON FUZZY CONTROL METHODS}

\section{K. Matrokhina}

Summary. The article discusses the method of fuzzy logical choice for solving the problem of choosing the best alternative. A formal model of the problem of multicriteria decision making and a decision algorithm are presented.

Keywords: fuzzy logic, decision support system, multicriteria choice methods, membership functions.

\author{
Матрохина Кристина Васильевна \\ Аспирант, Санкт-Петербургский горный \\ университет \\ k.matrokhina@mail.ru
}

Аннотация. В статье рассмотрен метод нечеткого логического выбора для решения задачи выбора лучшей альтернативы. Представлена формальная модель задачи многокритериального принятия решений и алгоритм решения.

Ключевые слова: нечеткая логика, система поддержки принятия решений, методы многокритериального выбора, функции принадлежности.

множества $A_{j}$ являются значениями лингвистической переменной $P$.

Пусть множество решений характеризуется набором критериев $p_{1}, p_{2}, \ldots, p_{n}$, т.е. лингвистических переменных, заданных на базовых множествах $u_{1}, u_{2}, \ldots, u_{p}$ соответственно. Набор из нескольких критериев с соответствующими значениями характеризует лица, принимающего решение, об оптимальности альтернативы. Высказывание может иметь следующий вид:

$$
\begin{aligned}
& b_{1}: \text { Eсли } p_{1}=A_{1}, u \quad p_{2}=A_{2 i} \quad u \ldots p_{n}=A_{n i} \\
& m o \quad S=B_{i}
\end{aligned}
$$

Аналогично, выражения $d_{1}, d_{2}, \ldots d_{q}$ преобразуются в множества $H_{1}, H_{2}, \ldots H_{q}$, а их пересечение является множеством $D$ :

$$
\mathrm{D}=H_{1} \cap H_{2} \cap \ldots \cap H_{q}
$$

и для каждого $(w, i) \in W x I$

$$
\mu_{D}(w, i)=\min _{w \in W}\left(\mu_{H_{i}}(w, i)\right), \quad j=\overline{1, q}
$$

Удовлетворительность альтернативы рассчитывается по следующей формуле:

$$
G=A \times D ，
$$

где $G$ - нечеткое подмножество интервала $I$.

\section{Тогда}


Таблица 1. Исходные данные

\begin{tabular}{|c|c|c|c|}
\hline \multirow[b]{2}{*}{ Критерий } & \multicolumn{3}{|l|}{ Альтернатива } \\
\hline & Моб.Интернет & Дом.Интернет & Wi-Fi TB \\
\hline$P_{1}-$ цена, руб/мес & 890 & 900 & 950 \\
\hline$P_{2}$ - качество, (1...5) & 4 & 5 & 3 \\
\hline$P_{3}-$ Спрос клиентов, $\%$ & 90 & 77 & 50 \\
\hline$P_{4}-$ разнообразие тарифов,(1 ...5) & 4 & 4 & 3 \\
\hline $\begin{array}{l}P_{5} \text { - наличие тех поддержки и качество } \\
\text { работы (1 ...5) }\end{array}$ & 5 & 4 & 3 \\
\hline
\end{tabular}

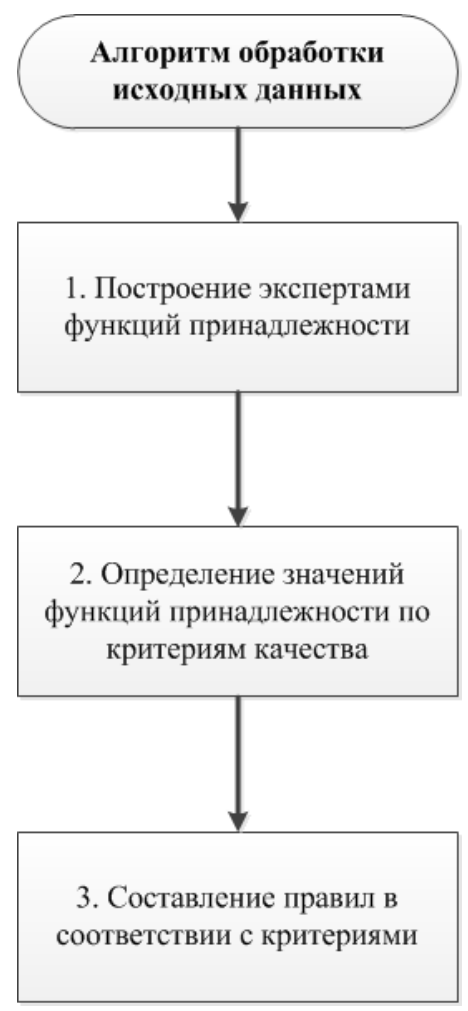

Рис. 1. Алгоритм обработки исходных данных

$$
\left.\mu_{G}(i)=\max _{w \in W}\left(\min \mu_{A}(w), \mu_{D}(w, i)\right)\right)
$$

Сопоставление альтернатив происходит на основе точечных оценок. Для нечеткого множества $E \subset I$ определено $\alpha-$ множество $(\alpha \in[0,1])$ :

$$
E_{\alpha}=\left\{i \mid \mu_{E}(i) \geq a \in I\right\}
$$

Точечное значение для множества С можно записать в виде:

$$
F(E)=\frac{1}{\alpha \max } \int_{0}^{\alpha \max } M\left(E_{\alpha}\right) d \alpha
$$

где $\alpha_{\max }-$ максимальное значение в множестве $Y$.

При сравнении альтернатив, сравниваются их точечные оценки и лучшей признается альтернатива с максимальным значением.

\section{Результаты}

Описанный метод позволяет повысить обоснованность принимаемых решений и обеспечить выбор наиболее оптимального варианта из множества допустимых. Задача выбора оптимальной услугу для продвижения компанией сформулирована следующим образом: телекоммуникационной компании требуется 
Таблица 2

\begin{tabular}{|l|l|l|l|l|l|l|l|}
\hline № & Условие & $P_{1}$ & $P_{2}$ & $P_{3}$ & $P_{4}$ & $P_{5}$ & $R$ \\
\hline 1 & Если & доступная & & высокий & большое & сверхрентабельная \\
\hline 2 & Если & завышенная & & & низкий & неоперативная & низкорентабельная \\
\hline 4 & Если & доступная & высокое & низкий & низкий & среднерентабельная & высокорентабельная \\
\hline 5 & Если & доступная & высокое & высокий & низкий & неоперативная & среднерентабельная \\
\hline 6 & Если & доступная & & низкий & & неоперативная & низкорентабельная \\
\hline
\end{tabular}

\begin{tabular}{|c|c|c|c|c|c|c|c|c|c|c|c|c|}
\hline \multirow{4}{*}{$D=$} & & 0 & 0,1 & 0,2 & 0,3 & 0,4 & 0,5 & 0,6 & 0,7 & 0,8 & 0,9 & 1 \\
\hline & $u_{1}$ & 0,5 & 0,5 & ого & 0,5 & 0,5 & 0,5 & 0,5 & 0,5 & 0,5 & 0,5 & 1 \\
\hline & $u_{2}$ & 0,4 & 0,4 & 0,4 & 0,4 & 0,4 & 0,4 & 0,4 & 0,4 & 0,4 & 0,4 & 0,8 \\
\hline & $u_{3}$ & $\| 0,45$ & 0,55 & 0,55 & 0,55 & 0,55 & 0,55 & 0,55 & 0,55 & 0,55 & 0,55 & 0,65 \\
\hline
\end{tabular}

Рис. 2

выбрать услугу, в которую в настоящий момент нужно вложить большее количество средств для продвижения ее на рынке и получения на выходе максимальной выгоды. В качестве альтернатив $\left(a_{1} \ldots a_{3}\right)$ рассматривается три типа тарифов: «Мобильный интернет», «Домашний интернет» и «Домашний интернет +ТВ»

Для осуществления выбора тарифа использованы маркетинговые данные компании (табл. 1).

Алгоритм обработки исходной информации включает в себя три этапа с применением метода нечеткого логического вывода (см.рис.1).

Нечеткие множества представлены следующим образом:

$$
\begin{aligned}
& \mu_{F_{1}}=0,5 / 890+0,6 / 900+0,45 / 950 ; \\
& \mu_{F_{2}}=0,8 / 4+1 / 5+0,65 / 3 ; \\
& \mu_{F_{3}}=0,85 / 90+0,7 / 77+0,55 / 50 ; \\
& \mu_{F_{4}}=0,85 / 4+0,8 / 4+0,45 / 3 ; \\
& \mu_{F_{5}}=1 / 5+0,8 / 4+0,65 / 3 .
\end{aligned}
$$

После проведения анализа экспертами были составленыследующиеправила: $P_{1}$ - цена; $P_{2}$ - качество; $P_{3}-$ спрос клиентов; $P_{4}$ - разнообразие тарифов; $P_{5}-$ наличие технической поддержки, $R$ - услуга (выходная).

Для того чтобы сформулировать правила, необходимо определить возможные значения лингвистических переменных $P_{i}$ и $R$, которые используются для оценки рентабельности услуги.

Область значений переменной $R$ задана на множестве $Y=\{0 ; 0,1 ; 0,2 ; \ldots ; 1\}$.
Значения переменной $R$ заданы с помощью следующих функций принадлежности:

$$
\begin{aligned}
& L S=\text { низкорентабельная, определена как } \\
& \mu_{L S}(x)=\left\{\begin{array}{l}
1, \text { если } \mathrm{x}=1 ; \\
0, \text { если } \mathrm{x}<1 .
\end{array} \text { х } \in \mathrm{J} ;\right. \\
& M S=\text { среднерентабельная - как } \\
& \mu_{M S}(x)=x, \mathrm{x} \in \mathrm{J} ; \\
& H S=\text { высокорентабельная - как } \\
& \mu_{H S}(x)=\sqrt{x} ; \mathrm{x} \in \mathrm{J} ; \\
& V S=\text { сверхрентабельная - как } \\
& \mu_{V S}(x)=1-x ; \mathrm{x} \in \mathrm{J} .
\end{aligned}
$$

Задача сводится к сравнению трех альтернатив (услуг) на множестве $U=\left\{u_{1}, u_{2}, u_{3}\right\}$.

Для данной задачи оценки трем альтерантивам заданы следующими нечеткими множествами: доступная (цена) $A=\left\{0,5 u_{1}, 0,6 u_{2}, 0,45 u_{2}\right\}$; высокое (качество) $B=\left\{0,8 u_{1}, u_{2}, 0,65 u_{3}\right\} ;$ высокий (спрос клиентов) $C=\left\{0,85 u_{1}, 0,7 u_{2}, 0,55 u_{3}\right\} ;$ большое (разнообразие тарифов) Д $=\left\{0,85 u_{1}, 0,8 u_{2}, 0,45 u_{3}\right\}$; оперативная (техническая поддержка) $E=\left\{u_{1}, 0,8 u_{2}, 0,65 u_{3}\right\}$.

Далее вычисляются функции принадлежности $\mu_{M_{1} \ldots M_{6}}$ для приведенных правил. 
На основании проведенных исследований, опубликованных в [17] в задачах рассматривающих определенный набор критериев по которым выбирается лучший вариант, как правило используют импликацию Лукасевича

$$
\mu_{D}=\min \left(1,1-\mu_{A} /(u)+\mu_{B}(j)\right)
$$

Для каждой пары значений получены нечеткие отношения $\left(D_{l}, \ldots, D_{6}\right)$.

Согласно импликации Лукасевича пересечения отношений $D_{1}, \ldots, D_{6}$ решение можно записать в виде следующей матрицы (рис. 2).

Для вычисления значений каждой альтернативы используется точечная оценка.

1) $H_{1}=\{0,5 / 0 ; 0,5 / 0,1 ; 0,5 / 0,2 ; 0,5 / 0,3 ; 0,5 / 0,4 ; 0,5 / 0,5$; 0,5/0,6;0,5/0,7;0,5/0,8;0,5/0,9; 1/1\} вычислены уровневые множества $H_{j a}$ и мощность такого множества $M\left(H_{a}\right)$ по формуле и точечная оценка $E_{l}$.

$$
F\left(H_{1}\right)=\frac{1}{\alpha \max } \int_{0}^{\alpha \max } M\left(H_{1 \alpha}\right) d \alpha=0,4^{*} 0,5+0,5^{*} 1=0,7
$$

2) $H_{2}=\{0,4 / 0 ; 0,4 / 0,1 ; 0,4 / 0,2 ; 0,4 / 0,3 ; 0,4 / 0,4 ; 0,4 / 0,5$; $0,4 / 0,6 ; 0,4 / 0,7 ; 0,4 / 0,8 ; 0,4 / 0,9 ; 1 / 1\}$ точечная оценка $H_{2}$ :

$$
F\left(H_{2}\right)=\frac{1}{\alpha \max } \int_{0}^{\alpha \max } M\left(H_{2 \alpha}\right) d \alpha=0,5^{*} 0,4+0,4^{*} 0,8=0,52
$$

3) $H_{3}=\{0,45 / 0 ; 0,55 / 0,1 ; 0,55 / 0,2 ; 0,55 / 0,3 ; 0,55 / 0,4$; $0,55 / 0,5 ; 0,55 / 0,6 ; 0,55 / 0,7 ; 0,55 / 0,8 ; 0,55 / 0,9 ; 0,65 / 1\}$ точечная оценка $H_{3}$ :

$$
\begin{aligned}
& F\left(H_{3}\right)=\frac{1}{\alpha \max } \int_{0}^{\alpha \max } M\left(H_{3 \alpha}\right) d \alpha= \\
& =0,45^{*} 0,5+0,5^{*} 0,1+0,1^{*} 1=0,375 .
\end{aligned}
$$

В качестве лучшей альтернативы выбирается та, которая имеет наибольшую оценку. Из полученных результатов можно заметить, что это альтернатива $u_{l}$.

\section{Выво $\Delta ы$}

При решении задачи метод нечеткого логического вывода показал свою эффективность в решении задач выбора лучшего варианта. Применение данного метода позволило выбрать лучшую услугу «Мобильный интернет» для ее продвижения компанией на телекоммуникационный рынок.

\section{ЛИТЕРАТУРА}

1. Чернов В.Г. Модели поддержки принятия решений в инвестиционной деятельности на основе аппарата нечетких множеств. -М.: Горячая линия-Телеком, 2007.-312 с., ил.

2. Зайнашева Э.Б., Мухаметзянов И.З. Модель взаимодействия конкурирующих фирм и принятие решений на основе аппарата нечеткой логики // Вестник УГНТУ. Наука, образование, экономика. Серия: Экономика. 2013. № 3 (5). URL: https://cyberleninka.ru/article/n/model-vzaimodeistviyakonkuriruyuschih-firm-i-prinyatie-resheniy-na-osnove-apparata-nechetkoy-logiki (дата обращения: 03.11.2021).

3. Карелова 0.Л. Методы поддержки принятия решений. Изд.: Проспект, 2020 г., с. 114.

4. Терегулов, Д.Р. Система поддержки принятия решений на основе метода учета трансформации предпочтений / Д.Р. Терегулов, 0.И. Сентябов // Информатика: проблемы, методология, технологии: материалы XV международной научно-методической конференции, Воронеж, 12-13 февраля 2015 года. — Воронеж: Воронежский государственный университет, 2015.— - P. 142-146.

5. Pavlov, S.V. Model of a fuzzy dynamic decision support system / S.V. Pavlov, V.A. Dokuchaev, S.S. Mytenkov // T-Comm. — 2020.— Vol. 14.— No 9.— P. 4347.- D OI 10.36724/2072-8735-2020-14-9-43-47.

6. Н.П. Резникова. Маркетинг в отрасли инфокоммуникаций /Кухаренко Е.Г.-М.: Горячая линия-Телеком, 2018.-152 с.: ил.

7. Nedosekin, Aleksei 0., Rejshahrit, Elena I., Kozlovskiy, Aleksandr N. (2019) Strategic approach to assessing economic sustainability objects of mineral resources sector of Russia. Journal of Mining Institute, vol. 237, pp. 354-360. D0I: 10.31897/PMI.2019.3.354

8. Н.В. Васильева, Э.Д. Кадыров (2013) Построение функций принадлежности параметров технологического процесса на основе нечеткой кластеризации производственных данных. Записки Горного института. Том 202. С. 251.

9. Isheyskiy, Valentin, Sanchidrián, José A. (2020) Prospects of applying MWD technology for quality management of drilling and blasting operations at mining enterprises. Minerals, issue 10, vol. 10, pp. 1-17. D0l: 10.3390/min10100925

10. Beloglazov, Ilia I., Boikov, Aleksei V., Petrov, Pavel A. (2020) Discrete element simulation of powder sintering for spherical particles. Key Engineering Materials, vol. 854 KEM, pp. 164-171. D0I: 10.4028/www.scientific.net/KEM.854.164

11. Pivovarova, I.I., Terekhin, R.D., Sarkisov, S.V., et al. (2020) Software implementation of fuzzy logic algorithms for environmental risk assessment. Journal of Physics: Conference Series, issue 2, vol. 1515, D0l: 10.1088/1742-6596/1515/2/022091

12. Малашенкова, И.В. Многокритериальные решения при объективных моделях в системах поддержки принятия решений / И.В. Малашенкова, Е.А. Панкратова, 0.В. Семенова // Естественные и технические науки. — 2014. — № 1(69). — C. 252-256.

13. Ефанов, Н.А. Сравнительный анализ существующих подходов к принятию управленческих решений в условиях неопределенности и систем поддержки принятия решений / Н.А. Ефанов // Экономика и управление: проблемы, решения.— 2020.— Т. 3.— № 2.— С. 129-137. 
14. Быкова Т.М. Системы поддержки принятия решений/ Соловьев А.Н. Изд.: Лань, 2020. - 132 с.

15. Л. Зааде. Понятие лингвистической переменной и его применение к принятию приближенных решений //М.: Мир, 1976. - 167 с.

16. Андрейчиков А.В. Анализ, синтез, планирование решений в экономике, г. Москва, «Финансы и статистика».2000. С. 210.

17. Knappe H., Nichtlineare Regelungstechnik und Fuzzy-Control. Expert Verlag. Renningen-Malmshein, Germany, 1994.

(c) Матрохина Кристина Васильевна ( k.matrokhina@mail.ru ).

Журнал «Современная наука: актуальные проблемы теории и практики»

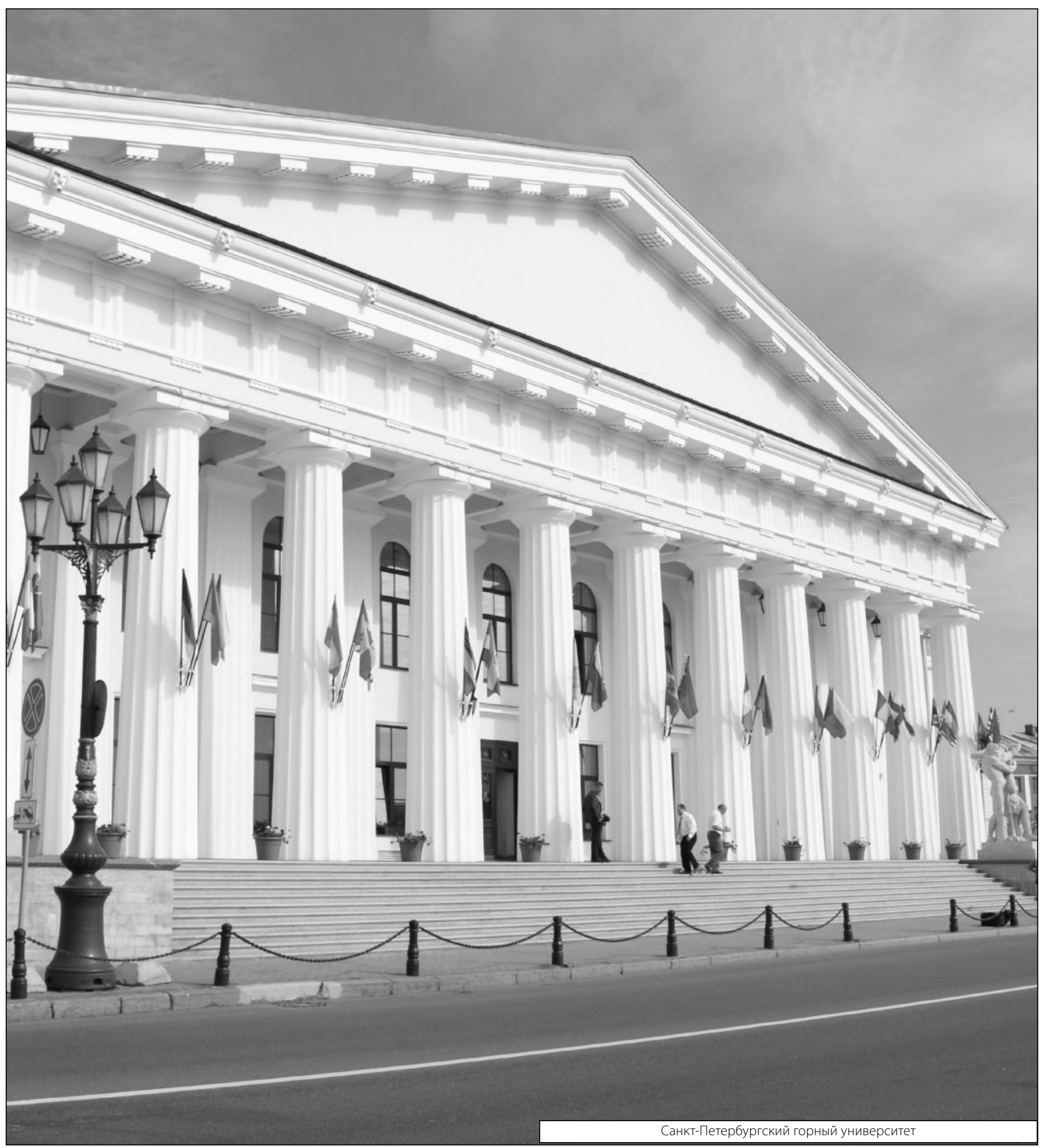

